\title{
Reconfiguration Performance of the Urban Power Distribution System Based on the Genetic-Ant Colony Fusion Algorithm
}

\author{
Longju Bai ${ }^{1, *}$ \\ ${ }^{1}$ College of Engineering, Beijing Forestry University, Beijing, China
}

\begin{abstract}
This study aims to enhance the reliability of the urban power grid system and decrease the economic loss due to power network faults. Based on the analysis of the traditional algorithms for restructuring the urban distribution system after faults, this study proposes an upgraded genetic algorithm (GA) and ant colony algorithm (ACA) and combines these two to overcome the limitations of the local optimum of GAs and low convergence speed of ACAs. Taking the IEEE33-node system as the research object, the network loss, maximum recovery of the power-loss load, and the number of switching operations as the objective function, the impact of different algorithms on the restoration and reconfiguration of the distribution system was examined according to MATLAB system simulation and the optimal algorithm for the reconfiguration of the urban distribution system failure recovery. The experimental results revealed that compared with the current distribution system reconfiguration algorithm, the genetic-ant colony algorithm (GACA) has higher algorithm time efficiency and solution accuracy and can markedly decrease the recovery time and improve the impact of the distribution system in a short period. Overall, the proposed GACA is an efficient self-healing algorithm of urban distribution systems and useful for augmenting the reliability of the urban power system.
\end{abstract}

\section{Introduction}

With the recent rapid growth of the economy and society, electric energy has progressively become an indispensable vital energy source. The power system comprises four parts-power generation, power transformation, power transmission, and power distribution. The distribution system directly connects with users and is a crucial part of the power system [1]. Unlike rural areas, cities are densely populated, economically developed, with a significant demand for education, transportation, and medical care; thus, they use more power and endure even shorter power outages. Besides the standard electric power distribution, the power distribution system is also one of the main tools for industrial development and optimal allocation, and is an integral part of the modern national economy and industrial development [2]. Thus, the power supply stability and security and reliability of power distribution systems are needed more strictly. Statistically, the average number of power outages due to the failure of medium voltage distribution systems accounts for approximately $80 \%$ of the total average number of power outages of users. When the power system fails, it is imperative to quickly decrease the scope and duration of power outages through effective fault recovery strategies [3], whereas it is challenging to decrease the probability of power outages only by reasonable planning of power network structure and installation of relay protection devices alone. The early distribution technology and management methods are primarily based on manual operation and experience, and the fault recovery of distribution systems is an extremely complex real-time decision-making process, which contains significant information. Under the trend of an increasingly complex structure of urban distribution systems, it is challenging for the dispatcher to decide on the spot in a short time according to the situation and experience, and it is easy to cause failure events that cannot be handled accurately and timely [4]. Thus, traditional power distribution technology and management methods can no longer produce breakthroughs in the development of distribution systems, nor can they fulfill various requirements of today's power distribution systems. Hence, how to choose new methods to handle the development of power distribution systems, enhance the self-healing ability of distribution systems, and increase the reliability of power systems has become a pressing scientific issue.

The urban power distribution system has the following characteristics: nonlinear, multiobjective, dynamic, and uncertain. In the problem of self-healing the distribution system, we should not only face the market uncertainty but also consider the uncertainty of the change of power load and the health of equipment [5]. Based on the characteristics of urban distribution systems, the power supply recovery of distribution systems fault is a multiobjective combinatorial optimization problem under allowable operation constraints and electrical constraints, which warrants fast, effective, reliable, and economical

* Corresponding author: longju bai@bjfu.edu.cn 
restoration of power supply in a fault-free power supply area based on the fault location and fault isolation while satisfying various constraints between load points [6]. At present, algorithms of the power distribution system reconfiguration are primarily divided into the following: mathematical optimization algorithm [7], heuristic algorithm [8], and artificial intelligence algorithm [9]. The artificial intelligence algorithm is extensively used in the power distribution system reconfiguration because of its advantages in managing nonlinear function relations. Atteya et al. [10] used binary coding of the artificial fish swarm algorithm in the power distribution system reconfiguration; although three simplification strategies were proposed, the issues of infeasible solution and dimension disaster were unavoidable. Another study [11] presented an enhanced differential evolution and simulated annealing hybrid algorithm for optimization calculation. To solve the problem that the traditional shortest path algorithm of flight path planning easily falls into the local optimal and complicated terrain, a method of flight path planning was proposed per the adaptive multistate fusion ant colony algorithm (ACA) [12], which avoided the local optimal solution effectively. Aiming at the nonlinear multiobjective optimization problem of the power distribution system reconfiguration, Jiao et al. [12] established a reconfiguration model of power distribution systems with distributed power supply, with the objectives of network loss, load balance of feeder, and optimal quality coordination of node voltage. Although numerous studies have proposed various improved algorithms to overcome the limitations of the algorithm itself, little research has been done to enhance the advantages of the algorithm itself. In the self-healing of the distribution system, many redundant iterations often occur, and the ability of model planning and self-healing is low, which results in the poor performance of the model.

Based on the analysis of the existing algorithms for the power distribution system restoration and reconfiguration, taking the IEEE33 node distribution system model as the research object, this study establishes a mathematical model to minimize network loss, minimizing the number of switching operations, and restoring the power-loss load as much as possible to upgrade ACA and genetic algorithm (GA), fuse the improved GA and ACA, and apply those proposed algorithms to the problem of the power distribution system restoration and reconfiguration. Through empirical analysis, this study provides a practical theoretical basis for the optimization of urban distribution systems and similar optimization problems.

\section{Methodology}

\subsection{Architecture and Fault Recovery of the Power Distribution System}

The power distribution system denotes the network of the low-voltage side of the secondary step-down substation in the entire power system, which primarily comprises overhead line, cable, pole tower, isolation switch, reactive power compensator, and equipment directly connected to the user. Usually, power distribution systems can be categorized into radial, tree, and ring types, as shown in Fig. 1. Under the requirement of a high-quality power supply, the automatic power distribution system comes into being, which mainly includes substation automation, feeder automation, load management, and distribution management automation [13].

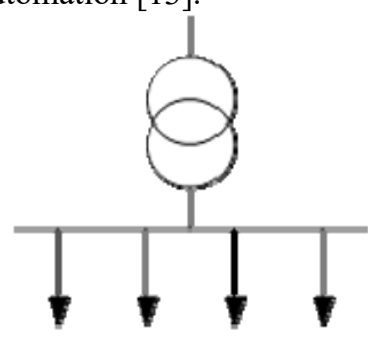

(a)

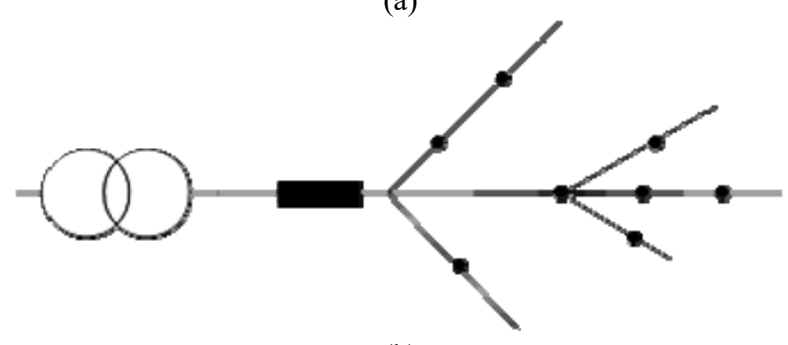

(b)

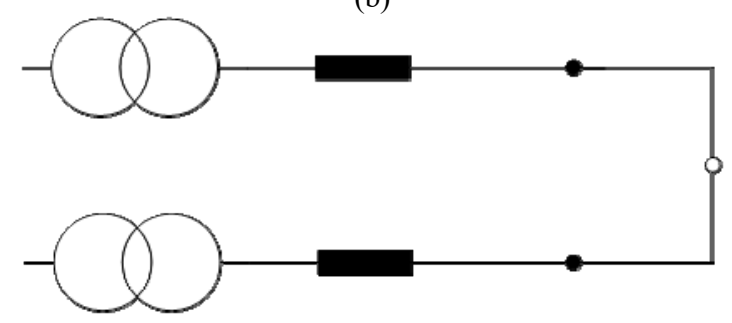

(c)

Figure 1. Connection mode of the distribution system.

(a) Radial system; (b) Tree system; (c) Ring system.

The power distribution system under $10 \mathrm{kV}$ typically has a closed-loop structure and open-loop operation. During standard operation, high-voltage transmission networks step down through transformers to supply power to a radial or tree-shaped power supply area. For separate power lines, a manual or automatic normally connected switch is usually used; however, for different power lines, a regular contact switch is used. Changes to these switches occur only when a line failure occurs, and the topology and operation of the entire system changes when the switching state of these switches is altered [14]. The power distribution system has the characteristics of closed-loop design and open-loop operation. The distribution system in different applications has different requirements for restoration and reconfiguration, for example:

1) Minimum network loss is as follows:

$$
f_{2}(x)=\sum_{k=1}^{n} i_{k}^{2} \cdot R_{k}
$$

where St denotes the actual load of elements; St max denotes the maximum allowable load of elements; and $n$ is the number of elements. 
2) Maximum recovery of the dead load is as follows:

$$
f_{3}(L)=\sum_{R} L_{k} y_{k}
$$

where $\mathrm{R}$ denotes the quantity of out-of-power load; Lk is the load of power line $\mathrm{k}$ in Lk non-fault power-loss area; and $\mathrm{yk}$ is the variable parameter (1: restored; 0 : unrecovered).

3) The number of operating a switch: many switches in the distribution network need manual operation by staff, which cannot guarantee the action speed and correct rate. Besides, the service life of switches is affected by the number of operation of switches; the more the operation, the shorter the switch life. Hence, it is essential to operate the switch as few times as possible.

$$
K=\sum_{i=1}^{N}\left|k_{i}-k_{i}^{\prime}\right|
$$

where $\mathrm{N}$ denotes the number of switches in the power-off area; $\mathrm{K}$ is the switch state (1: close, 0 : open).

Constraints:

1) Power flow constraint of distribution networks

$$
\begin{gathered}
P_{m(i)}=\sum P_{H(i)}+\sum_{m \in D_{i}}\left(P_{(i m)}+P_{L(i m)}\right) \\
Q_{m(i)}=\Sigma Q_{H(i)}+\sum_{m \in D_{i}}\left(Q_{(i m)}+Q_{L(i m)}\right)
\end{gathered}
$$

where Di denotes the set of outgoing lines connected on node i; Pm (i) denotes the active power injected into node $\mathrm{i}$; $\sum \mathrm{PH}$ (i) is the active load of node $\mathrm{i}$; $\mathrm{P}$ (im) is the active power flowing through the m-branch of node $\mathrm{i}$; PL (im) is the active power loss on the $\mathrm{m}$-branch of node $i$.

2) Line capacity constraint

$$
S_{i} \leq S_{i \max }
$$

3) Line current constraint

$$
I_{i j} \leq I_{i j \max }
$$

4) Bus voltage constraint

$$
V_{i \min } \leq V_{i} \leq V_{i \max }
$$

5) Radial constraints of distribution networks

$$
g_{k} \in G_{K}
$$

where gk denotes the current network structure; and Gk is the set of all allowable radial network configurations.

\subsection{Principle and Improvement of Genetic Algorithm}

GA transforms the process of solving the problem into the process of crossing and mutation of chromosome genes in biological evolution, and finally converges to the population of "most suitable environment," to obtain the optimal solution to the problem. Many problems exist in applying GA in power distribution system planning, among which the crossover operator can retain good genes; however, it is easy to lead to a premature algorithm. Although the mutation operator can solve this problem to some extent, the mutation probability is usually small, and it is challenging to jump out of the local optimum. For distribution system planning, connectivity and radial constraints are the most crucial factors that affect the feasibility of the solution, and the crossover operator of
GA can easily destroy the feasibility of the solution. Producing a large number of infeasible solutions decreases search efficiency. Aiming at the issue that the traditional GA has limited searching space and easily falls into the local optimal solution during optimization, this study introduced an improved GA [15], in which a single population is extended to many groups, and the Metropolis criterion in the simulated annealing algorithm is introduced to determine whether new individuals are accepted or not. Figure 2 shows its basic process.

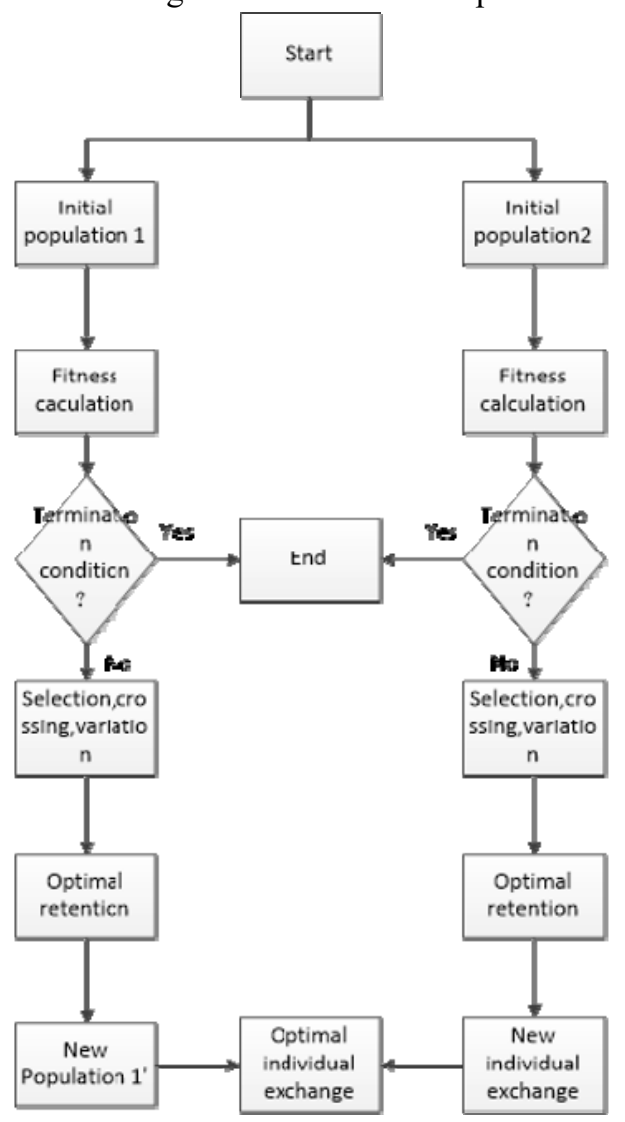

Figure 2. Flow diagram of the double population genetic algorithm.

Before using GA, the actual problem must be transformed into machine-identifiable coding, that is, the problem of optimization must be addressed. As binary coding takes up a great deal of memory, and increases the cost of computing resources, Gray code coding was used in this study. The specific method is as follows:

$$
Y=\left\{\begin{array}{l}
y_{m}=x_{m} \\
y_{i}=x_{i+1}, i=m-1, \ldots, 1
\end{array}\right.
$$

where $\mathrm{X}$ is binary coding. The selection of GA is to select the suitable operator from SGA, usually using Monte Carlo operator. For each individual, the correlation between fitness and total fitness is as follows:

$$
F_{s}=F\left(x_{1}\right)+F\left(x_{2}\right)+K+F\left(x_{N}\right)
$$

At this point, a random number is generated on $F_{s}$, following which the fitness of all individuals is accumulated from the first individual. When the cumulative value exceeds, the last individual of $F_{s}$ becomes the selected individual. In this study, we used 
single-point cross-operation, and mutation recombined the existing patterns in the population; thus, we continued to enhance the search ability of the population. The variation method is as follows:

First, the mutation probability $p_{m}$ was set, and then a random number $r_{i}$ was generated for each gene in a chromosome string using a random function Rand () consistent with uniform distribution $U(0,1)$. The gene value corresponding to the encoding position varies. When chromosomes are strung as $\mathrm{s}$, then:

$$
\alpha=\left\{\begin{array}{l}
1-\alpha_{i}, r_{i}<p_{m} \\
\alpha_{i}, r_{i} \geq p_{m}
\end{array}\right.
$$

Individuals selected, crossed, and mutated should be examined for their fitness. High fitness implies that individuals should be more reserved. The fitness function must satisfy the non-negative characteristic. The fitness function $\mathrm{F}()$ used in this study is as follows:

$$
F\left(x_{i}\right)=\frac{N-i+1}{N}, i=1,2 \ldots, N
$$

To address the optimization problem, the iterative termination condition must be provided for the algorithm. The termination condition of this paper is as follows:

$$
\left|f\left(x_{\text {text }}^{\prime}-f(x)^{\prime}\right)\right| \leq \varepsilon
$$

where, $f\left(x_{\text {text }}^{\prime}\right)$ denotes the optimal individual in the generation; $f(x)^{\prime}$ denotes the optimal solution of the objective function of the problem; and $\varepsilon$ is the required solution precision. The calculations for the Metropolis criteria are as follows:

$$
P(i->j)=\left\{\begin{array}{l}
1, f(j) \leq f(i) \\
e^{\frac{f(i)-f(j)}{t}}, f(j)>f(i)
\end{array}\right.
$$

where $\mathrm{t}$ denotes the temperature control parameter in simulated annealing algorithm, which decreases with the increase of iteration times. The introduction of this criterion renders the population search not only in a favorable direction but also in a poor direction. Accordingly, the individual's search ability was improved, and the local optimal solution was avoided.

\subsection{Principle and Improvement of Ant Colony Algorithm}

ACA is a heuristic search algorithm based on population optimization and a bionic algorithm to search the shortest path from nest to food. It has a positive feedback acceleration algorithm evolution process, and distributed collaboration makes ACA easy to attain parallel, which improves the communication and transfer of information between individual ants to avoid local optimization. However, the search efficiency of the basic ACA is low, and the search quality is poor. This study adopted the best - the worst ant system. The algorithm uses the antweek system, which releases pheromones after the ants have established a complete trajectory, using the whole information [16]. The improved algorithm primarily modifies the global update formula in the ant colony system. The specific steps are as follows:
(1) initialization;

(2) select a path for each ant according to the following formulas:

$$
P_{i j}^{k}(t)=\left\{\begin{array}{c}
\frac{\tau_{i j}^{\alpha}(t) \eta_{i j}^{\beta}(t)}{\sum_{m \in N_{k}} \tau_{i m}^{\alpha}(t) \eta_{i m}^{\beta}(t)}, m \in N_{k} \\
0, \quad \text { others }
\end{array}\right.
$$

and

$$
\tau_{i j}(t+n)=\rho_{1} \cdot \tau_{i j}(t)+\Delta \tau_{i j}(t, t+n)
$$

where,

(3) execute local update with $\tau(r, s)-(1-\rho) \cdot \tau(r, s)+\rho \cdot \Delta \tau(r, s)$ when the path of every ant is generated;

(4) iterate steps (2) and (3) until each ant generates a path;

(5) choose the best and the worst ants;

(6) execute global update rules for the best ants with the following formulas:

$$
\begin{gathered}
\tau(r, s)-(1-\alpha) \cdot \tau(r, s)+\alpha \cdot \Delta \tau(r, s) \\
\Delta \tau(r, s)= \begin{cases}\left(L_{a b}\right)^{-1} & \text { if }(r, s) \in \text { Global optimal paths } \\
0 & \text { otherwise }\end{cases}
\end{gathered}
$$

where $\alpha$ denotes the volatilization coefficient of pheromone, $0<\alpha<1 ; L_{a b}$ denotes the global optimal path so far. This formula stipulates that only pheromones on the edge of the global optimal path will be enhanced;

(7) execute the global update rule for the worst ants per the following formula:

$$
\tau(r, s)-(1-\rho) \cdot \tau(r, s)-\varepsilon \cdot \frac{L_{w o r s t}}{L_{\text {best }}}
$$

where $\varepsilon$ is a parameter introduced in the algorithm; L_worst represents the path length of the worst ant in the current cycle; L_best represents the path length of the best ant in the current cycle; $\tau(\mathrm{r}, \mathrm{s})$ denotes the pheromone trajectory between city $\mathrm{r}$ and city $\mathrm{s}$.

(8) iterate steps (2)-(7) until the number of executions reached the specified number or there is no better solution for several successive generations.

Figure 3 shows the specific flowchart of the ant colony algorithm improvement. 


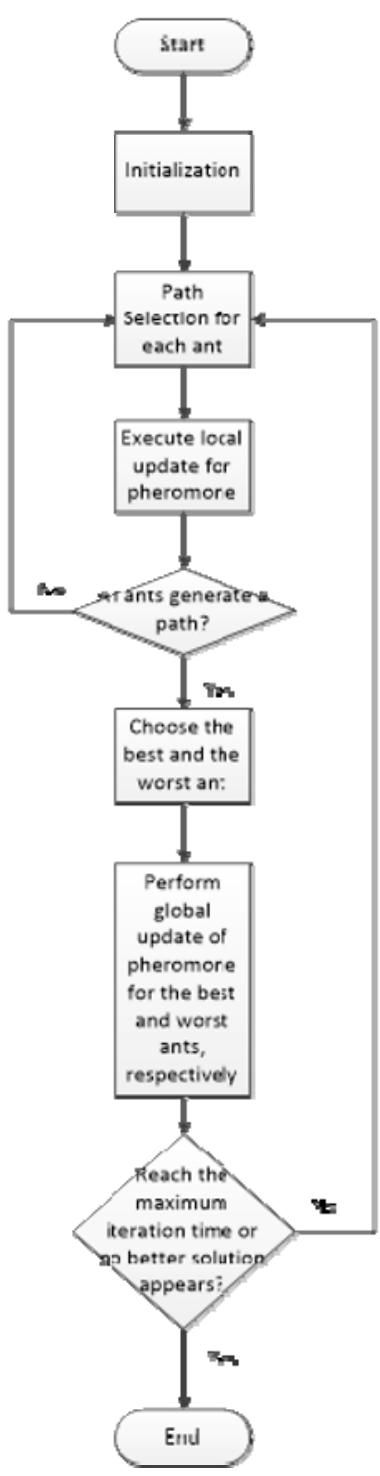

Figure 3. Flowchart of the ant colony algorithm improvement.

\subsection{Genetic-Ant Colony Fusion Algorithm}

GA has an excellent performance in global searching ability; however, it often ignores feedback information affecting the solving speed. ACA searching the highquality solution of the problem by accumulating pheromone; however, there is no pheromone in the initial stage of searching optimization, which makes the algorithm less efficient. Both algorithms are probabilistic optimization and do not rely on the strict mathematical properties (continuity, conductivity, differentiability) of the problem itself and the precise mathematical model of the variable function. In this study, the two algorithms were combined to solve the problem, and the fast global searching ability of GA was used to provide the required pheromone for ACA to solve the problem, which could complement each other and attain the win-win of optimization performance and time performance.

The following steps ensured that the GA and ACA were fused at the best time. First, set the minimum number Gene ${ }_{\min }$ of genetic iterations and the maximum number Gene $_{\max }$ of genetic iterations. Second, in the process of genetic iteration, the population similarity difference $\Delta K$ between two adjacent generations was counted, and the minimum evolution rate $\Delta K_{\min }$ of the progeny population was set accordingly. Finally, within the set number of iterations, if successive $\operatorname{Gen} \theta_{\text {die }}$

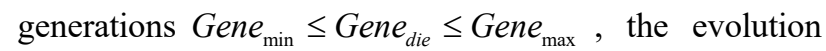
rate of the offspring population was less than $\Delta K_{\min }$, indicating the GA optimization speed was low, the GA process can be terminated and the ACA can be entered.

The top $10 \%$ of individuals with the best fitness value in the population at the termination of the GA were selected as the genetic optimization solution set, which was recorded as $S_{10 \% \text { better }}^{\text {gene }}$ for pheromone conversion. The initial value of the pheromone was $\tau_{S}=\tau_{C}+\tau_{G}$, where $\tau_{c}$ denotes a pheromone constant given according to the scale of the problem to be solved, $\tau_{a}$ signifies the pheromone value converted from the result of the GA, and $\tau_{G}=Q \times f_{\text {best }}(x)$, and $\mathrm{Q}$ is a constant. Figure 4 shows the specific process of the genetic-ant colony algorithm.

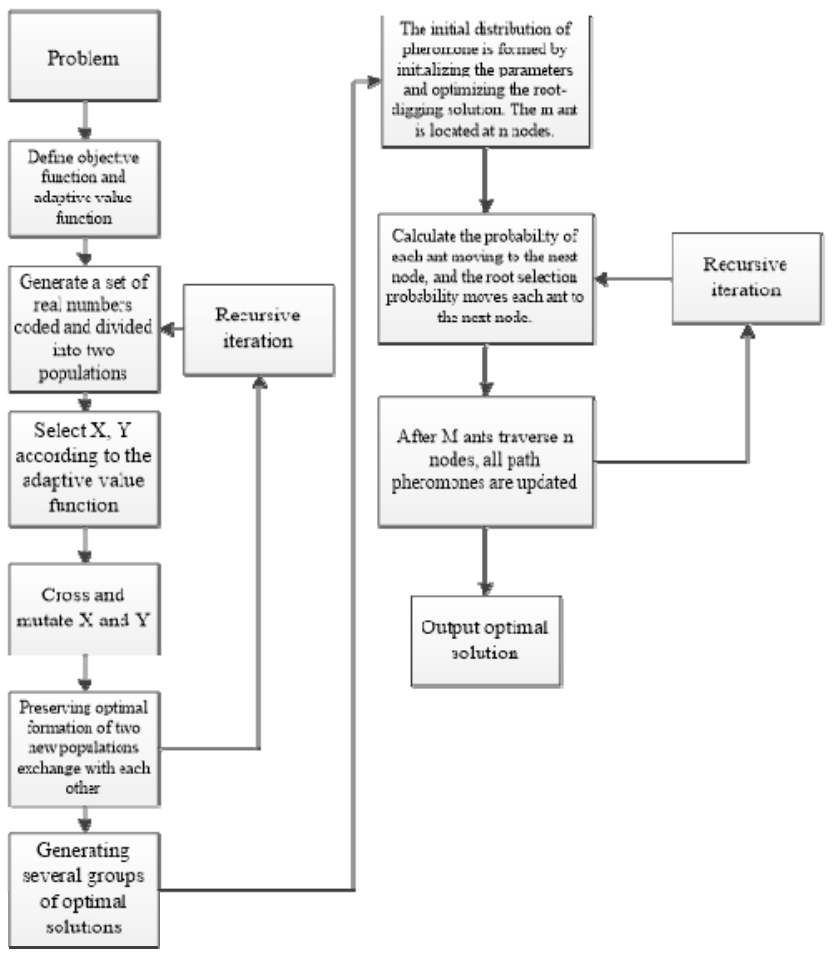

Figure 4. Flowchart of the genetic-ant colony algorithm.

\subsection{Data Input and Parameter Determination}

(1) Data Set: In this study, 48 city TSP problem (Att48) and 51 city TSP problem (Eil51) were used to test the algorithm model. The problem was from TSP standard problem library (TSPLIB) with the following parameter: 
Table 1. Att48 parameter settings

\begin{tabular}{|c|c|c|c|c|c|}
\hline 167341453 & 2223310 & 355301424 & 4401841 & 530821644 & 676084458 \\
\hline 775733716 & 872651268 & 968981885 & 1011122049 & 1154682606 & 1259892873 \\
\hline 1347062674 & 1446122035 & 1563472683 & 166107669 & 1776115184 & 1874623590 \\
\hline 1977324723 & 2059003561 & 2144833369 & 2261011110 & 2351992182 & 2416332809 \\
\hline 2543072322 & 266751006 & 2775554819 & 2875413981 & 293177756 & 3073524506 \\
\hline 3175452801 & 3232453305 & 3364263173 & 3446081198 & 35232216 & 3672483779 \\
\hline 3777624595 & 3873922244 & 3934842829 & 4062712135 & 414985140 & 4219161569 \\
\hline 4372804899 & 4475093239 & 45102676 & 4668072993 & 4751853258 & 4830231942 \\
\hline
\end{tabular}

Table 2. Eil51 parameter settings

\begin{tabular}{|c|c|c|c|c|c|}
\hline 13752 & 24949 & 35264 & 42026 & 54030 & 62147 \\
\hline 71763 & 83162 & 95233 & 105121 & 114241 & 123132 \\
\hline 13525 & 141242 & 153616 & 165241 & 172723 & 181733 \\
\hline 191313 & 205758 & 216242 & 224257 & 231657 & 24852 \\
\hline 25738 & 262768 & 273048 & 284367 & 295848 & 305827 \\
\hline 313769 & 323846 & 334610 & 346133 & 356263 & 366369 \\
\hline 373222 & 384535 & 395915 & 4056 & 411017 & 422110 \\
\hline 43564 & 443015 & 453910 & 463239 & 472532 & 482555 \\
\hline
\end{tabular}

(2) Model parameters: The parameters of the algorithm were as follows: the crossover probability in GA was 0.85 , mutation probability was 0.12 , population number Att48 was 96, Eil51WEI was 102; ACA ant number Att48 was 32, Eil51WEI was 34, elicitation factor was 1 , expected elicitation factor was 10 , information retention degree was 0.5 , pheromone intensity was 120 ; TSP runs 30 times; artificial fish swarm algorithm: $\mathrm{Cli}=$ $0.155, \mathrm{C} 2 \mathrm{i}=0.05, \mathrm{tmaxi}=3000$, Asize $=30$, Visual $=5$, m1 Try-number $=6, \mathrm{~d}=0.25$.

(3) Simulation example: IEEE 33-node distribution system was selected as an example (as shown in Fig. 2-5). The whole system comprised 33 nodes, 37 branches, rated voltage $12.66 \mathrm{kV}$, the total load of the system of 3.802 $\mathrm{kW}$, branch impedance of $0.6 \Omega$, node load parameters (each load point parameter took the same value, load size of $350 \mathrm{kV} \mathrm{A}$, power factor of 0.9 , maximum load loss hours of $2800 \mathrm{~h}$, equipment life of 25). The power flow calculation method is as follows:

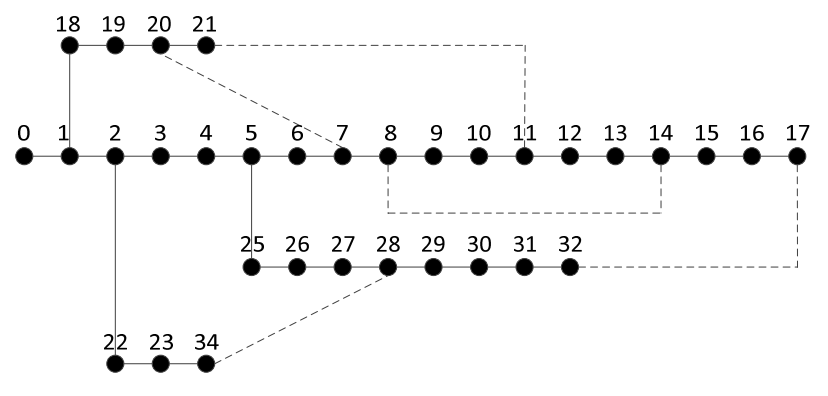

Figure 5. IEEE33 node distribution system.

As shown in Fig. 5, the mathematical model of the power line in the steady-state analysis of the power system is the equivalent circuit represented by resistance, reactance, susceptance, and conductance.

$$
r_{1}=\frac{\rho}{s}
$$

where $\rho$ denotes the resistivity of the conductor material $\left(\Omega \bullet \mathrm{mm}^{2} / \mathrm{km}\right)$; and $\mathrm{s}$ represents the rated cross-sectional area $\left(\mathrm{mm}^{2}\right)$ of the conductor.

$$
x_{1}=0.1445 \lg \frac{D_{m}}{r}+0.0157
$$

where $r$ is the calculated radius $(\mathrm{mm}$ or $\mathrm{cm}$ ) of line; and Dm denotes the geometric mean distance $(\mathrm{mm}$ or $\mathrm{cm})$, it should be the same unit as that of $r$.

$$
\begin{gathered}
b_{1}=\frac{7.58}{\lg \frac{D_{m}}{r}} \times 10^{-6} \\
g_{1}=\frac{\Delta P_{g}}{U^{2}} \times 10^{-3}
\end{gathered}
$$

where b1 denotes susceptance per unit length of conductor $(\mathrm{S} / \mathrm{km})$; g1 denotes conductance per unit length of conductor $(\mathrm{S} / \mathrm{km}) ; \Delta P_{g}$ denotes three-phase line leakage and corona loss power $(\mathrm{kW} / \mathrm{km})$; $\mathrm{U}$ is line voltage $(\mathrm{kV})$. According to the formula provided above, the resistance, reactance, susceptance, and conductance of a unit length conductor can be used as the original equivalent circuit diagram of the power line, as shown below. This is a single-phase equivalent circuit. Furthermore, one-phase equivalent circuit can be used to represent three-phase because the three-phase equivalent circuit is discussed in this study.

\section{Results and Discussion}

\subsection{Algorithm Model Comparison}

As shown in Table III, we applied the three algorithms mentioned above to compare two typical TSP problems. Table III shows that in the 48 cities TSP problem (Att48) and 51 cities TSP problem (Eil 51), the algorithms proposed in this study were small in search time and iteration times, among which the genetic-ant colony algorithm (GACA) had a less optimal solution and time on different problems. Among the three algorithms, the fusion algorithm could overcome the limitation that GA can easily fall into local optimal and ACA is slow to converge, and the optimal scheme can be obtained quickly and effectively for the typical optimization problem. Besides, the performance is the best. These experimental results suggested that the fusion GACA has a solid ability to find better solutions.

Table3. Comparison of three optimization algorithms for the TSP problem

\begin{tabular}{ccccccc}
\hline $\begin{array}{c}\text { Different } \\
\text { algorithms }\end{array}$ & \multicolumn{2}{c}{$\begin{array}{c}\text { Improved } \\
\text { genetic }\end{array}$} & \multicolumn{2}{c}{$\begin{array}{c}\text { Improved ant } \\
\text { colony }\end{array}$} & \multicolumn{2}{c}{$\begin{array}{c}\text { Genetic-Ant } \\
\text { Colony Algorithm }\end{array}$} \\
\hline Standard problem & Att48 & Eil51 & Att48 & Eil51 & Att48 & Eil51 \\
Optimal solution & 1024 & 482 & 1169 & 432 & 885 & 416 \\
Inferior solution & 1163 & 513 & 1246 & 498 & 893 & 435 \\
Average & 1093.5 & 497.5 & 1207.5 & 465 & 889 & 425.5 \\
Time/s & 99.4 & 46.24 & 257.3 & 123.6 & 85.7 & 32.4 \\
Optimum & 889 & 425.5 & & & & \\
\hline
\end{tabular}




\subsection{Performance Comparison of Different Models}

To evaluate and validate the accuracy and efficiency of the intelligent reconfiguration algorithm in this study, the algorithms were compared with the fault recovery reconfiguration algorithms proposed in recent years. Figure 6 presents a simulation image of network loss and iterations of the fault recovery reconfiguration under different algorithms. Table IV presents a comparison of each index in the simulation process. The graph indicates that the GACA in this experiment is better than the ACA, GA, and artificial fish colony algorithm in a short time and a small number of iterations

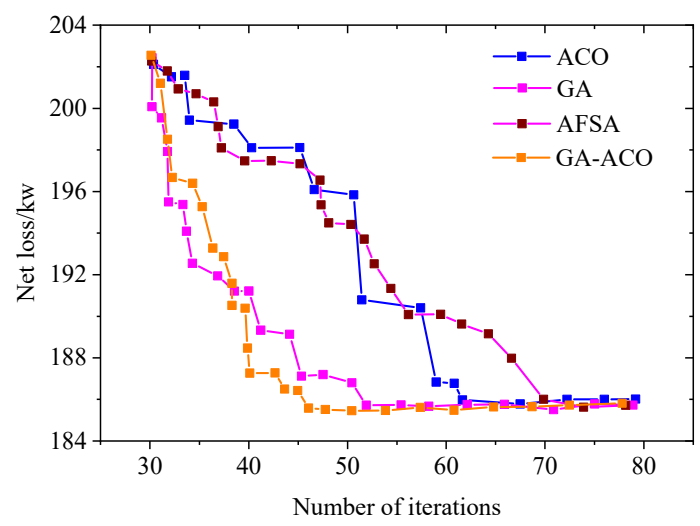

Figure 6. Iterative convergence curve.

Table 4. Performance comparison of different algorithms

\begin{tabular}{|c|c|c|c|c|}
\hline Algorithm & Net loss & $\begin{array}{c}\text { Maximum recovery } \\
\text { of dead load }\end{array}$ & $\begin{array}{c}\text { Number of } \\
\text { operating switches }\end{array}$ & $\begin{array}{c}\text { Average number } \\
\text { of iterations }\end{array}$ \\
\hline $\begin{array}{r}\text { Ant colony } \\
\text { algorithm }\end{array}$ & $152.6 \mathrm{~kW}$ & $300 \mathrm{~kW}$ & 25 times & 82.5 \\
\hline Genetic algorithm & $146.8 \mathrm{~kW}$ & $220 \mathrm{~kW}$ & 20 times & 80.3 \\
\hline $\begin{array}{l}\text { Artificial fish } \\
\text { swarm algorithm }\end{array}$ & $182.4 \mathrm{~kW}$ & $160 \mathrm{~kW}$ & 15 times & 96.3 \\
\hline $\begin{array}{c}\text { Genetic-Ant } \\
\text { Colony Algorithm }\end{array}$ & $142.4 \mathrm{~kW}$ & $450 \mathrm{~kW}$ & 26 times & 47.6 \\
\hline Time/s & 99.4 & 46.24 & 257.3 & 123.6 \\
\hline Optimum & 889 & 425.5 & & \\
\hline
\end{tabular}

\subsection{Model Parameter Optimization}

(1) Setting of parameters $\varepsilon$

The ACA part of the GACA adopts the improved best-worst ant system. To explore the utility of the bestworst ant system for each cycle performed by global updating formula, the simulation results obtained were compared by changing the parameter settings of $\varepsilon$. By changing the $\varepsilon$ value, the route taken by the worst ant can be weakened. The larger the $\varepsilon$ value, the more are weakened. The tested $\varepsilon$ values were respectively [0, 0.2 , $0.5,1.0,5.0,20.0,100.0]$. The optimal best-worst ant system restored to the ant colony system when $\varepsilon$ was set to 0 . The same was also performed in the IEEE 33-node distribution network model. A total of 30 cycles were run, and the average value of the experimental results was taken. Table $\mathrm{V}$ lists the simulation results. The experiments revealed that by properly changing parameter $\varepsilon$, the application of each loop ants performed worst global update formula to enhance the algorithm performance to accelerate its convergence speed.

Table 5. The simulation results for different $\varepsilon$

\begin{tabular}{|c|c|c|c|c|c|c|c|}
\hline${ }_{\varepsilon}$ Parameter & 0 & 0.2 & 0.5 & 1 & 5 & 20 & 100 \\
\hline Net loss & $215.6 \mathrm{~kW}$ & $206.9 \mathrm{~kW}$ & $196.3 \mathrm{~kW}$ & $142.4 \mathrm{~kW}$ & $152.4 \mathrm{~kW}$ & $163.6 \mathrm{~kW}$ & $172.4 \mathrm{~kW}$ \\
\hline $\begin{array}{l}\text { Maximum } \\
\text { recovery of } \\
\text { dead load }\end{array}$ & $150 \mathrm{~kW}$ & $219 \mathrm{~kW}$ & $365 \mathrm{~kW}$ & $450 \mathrm{~kW}$ & $412 \mathrm{~kW}$ & $425 \mathrm{~kW}$ & $436 \mathrm{~kW}$ \\
\hline $\begin{array}{l}\text { Number of } \\
\text { operating } \\
\text { switches }\end{array}$ & 36 times & 35 times & 20 times & 15 times & Ten times & 16 times & 21 times \\
\hline $\begin{array}{c}\text { Average } \\
\text { number of } \\
\text { iterations }\end{array}$ & 90.8 & 86.3 & 56.9 & 47.6 & 49.3 & 51.2 & 56.9 \\
\hline
\end{tabular}

(2) Setting of the parameter $\Delta K$

The difference $\Delta K$ of $\mathrm{K}$ value in different iterations of the population reflected the evolution speed of the population. If the difference of population similarity was less than the preset value, the GA could be terminated when the efficiency of iteration was decreased, and the fusion point was close to the best fusion point. The convergence efficiency of GA-ACO fusion algorithm was markedly influenced by a different value of $\Delta K$, and when the performance of GA-ACO fusion algorithm was the best in solving the efficiency and iterating number, the value of $\Delta K$ was 0.15 . Thus, this study took $\Delta K=0.15$.

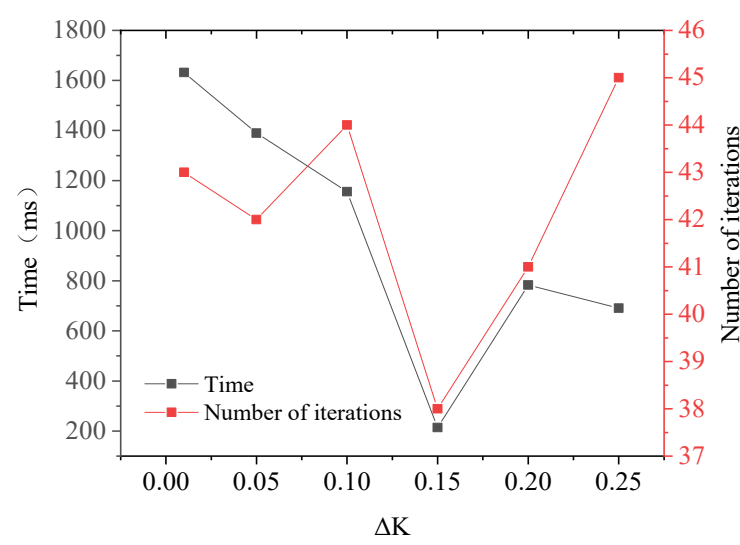

Figure 7. Settings of PARAMETERS $\Delta K$.

\subsection{Application Analysis of Model}

(1) As shown in Fig. 8, convergence occurred at the eleventh iteration. At the beginning of $n=45$, the target function value, that is, the network loss of the distribution network, remained unchanged, and the concentration of pheromones on each edge was not updated; thus, the optimal solution could be considered. The network loss of the distribution network was reduced to $99.58 \mathrm{~kW}$ with the original $139.6 \mathrm{~kW}$; thus, the hybrid algorithm of this study was effective in decreasing the network loss. 


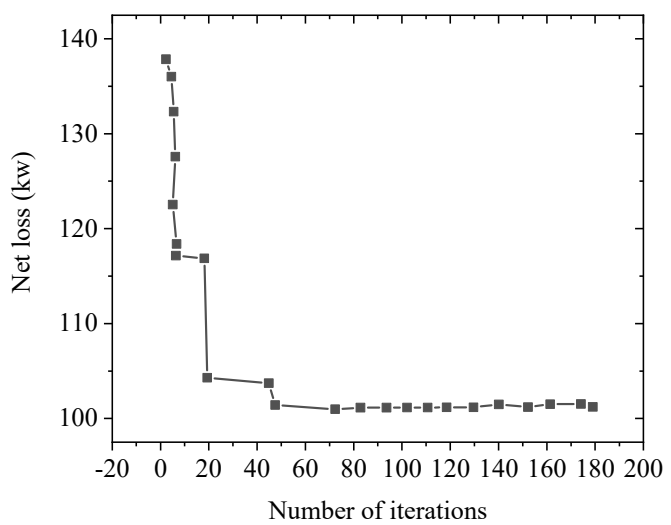

Figure 8. Variation of the target function value.

(2) Figure 8-9 shows the correlation between voltage and load margin of the distribution system before and after reconfiguration. Apparently, after the network reconfiguration, the control capability of the distribution network was enhanced if the voltage quality qualified. Before the reconfiguration, when the total load of the system increased to 1.1 times the original load, the lowest voltage of 46 nodes of the system was $<0.9$, lower than the maximum voltage limit for the system operation. The load control ability of the reconstructed system was markedly improved. When the load increased to $1.6 \mathrm{~L}$, the operation mode could still be carried out safely and effectively.

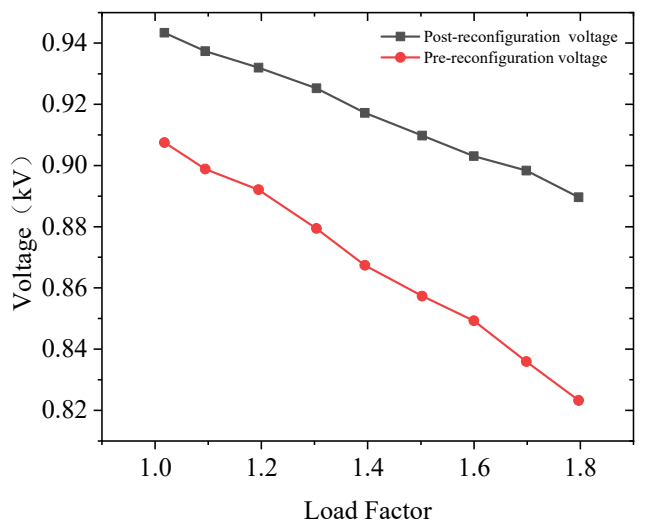

Figure 9. System voltage variation before and after reconfiguration when load increases.

(3) As shown in Fig. 10, the GACA was used to search the branches that should be segmented. After 30 experiments, the optimal solution can be converged every time. The convergent algebra was concentrated in the third to the fifth generation, only once in the sixth generation, and the calculation speed of this method was high.

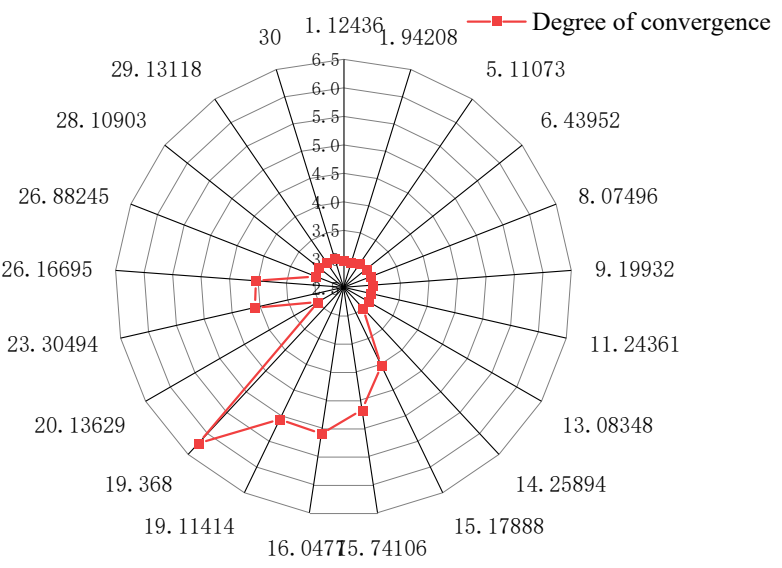

Figure 10. Simulation results.

(4) As shown in Table VI, for the same example, Azizivahed et al. [17] used fuzzy GA, the length of chromosome was 74 , the initial population was 100 , the initial crossover probability was 0.9 , the initial mutation probability was 0.01 , and the optimal solution was obtained when iterating to 300 generations. In addition, Azizivahed et al. [17] used improved GA, the length of chromosome was 59, the initial population was 50, the initial crossover probability was 0.9 , the initial mutation probability was 0.01 , and the optimal solution was obtained when iterating to 21 generations. The hybrid algorithm of this study improved the convergence speed.

Table 6. Performance comparison of different models

\begin{tabular}{cccc}
\hline & Pre-reconfiguration & Ref. [17] & This study \\
\hline & $5-10$ & $11-16$ & $6-9$ \\
Open switch set & $13-16$ & $13-10$ & $13-15$ \\
& $15-17$ & $14-19$ & $11-12$ \\
& $26-33$ & $17-28$ & $17-21$ \\
Network loss & $18-29$ & $21-33$ & $17-30$ \\
$(\mathrm{~kW})$ & 263.5 & 168.7 & 93.2 \\
Minimum & & & \\
voltage point & 0.8147 & 0.9245 & 0.9006 \\
\hline
\end{tabular}

\section{Conclusions}

Based on the analysis of the current urban power distribution system structure and fault recovery reconfiguration algorithm, this study introduces an upgraded GA and ACA to overcome the limitations of these two algorithms and enhance the performance of the algorithm. Compared with previous research, the GACA proposed in this study can be used to reconstruct the urban distribution network with less loss, larger maximum recovery, and fewer switch action times in a short time. In addition, this study can provide some ideas and methods for urban distribution network restoration and reconfiguration. Although this study effectively solves the problem of power distribution system restoration and reconfiguration, there remain many limitations. First, load data were the basis of power flow calculation of distribution system reconfiguration; however, the current 
distribution system cannot get accurate data of each load point. Thus, strengthening the accuracy of load forecasting, decreasing the error of load forecasting, and adopting the appropriate load model could help the distribution system reconfiguration to obtain the exact solution and make the distribution system reconfiguration practical as soon as possible. Second, for the large-scale distribution system, most optimization search algorithms cannot find the optimal solution and calculate the time in a good compromise. Hence, it is worth further investigation to find suitable algorithms for fusion and overcome the limitations.

\section{References}

1. Gunasekaran, M.,Mohamed Ismail, H.,Chokkalingam, B.,Mihet-Popa, L.,Padmanaban, S. Energy management strategy for rural communities' DC micro grid power system structure with maximum penetration of renewable energy sources. Applied Sciences. 2018, 8(4): 585-596.

2. Ehsan, A.,Yang, Q. State-of-the-art techniques for modelling of uncertainties in active distribution network planning: A review. Applied energy. 2019, 239: $1509-1523$

3. Khameneh, P.A.,Lavasani, S.M.,Nodehi, R.N.,Arjmandi, R. Water distribution network failure analysis under uncertainty. International Journal of Environmental Science and Technology. 2020, 17(1): 421-432.

4. Byrne, R.H.,Nguyen, T.A.,Copp, D.A.,Chalamala, B.R.,Gyuk, I. Energy management and optimization methods for grid energy storage systems. IEEE Access. 2017, 6: 13231-13260.

5. Morote, Á.-F.,Hernández-Hernández, M. Unauthorised domestic water consumption in the city of Alicante (Spain): A consideration of its causes and urban distribution (2005-2017). Water. 2018, 10(7): 851-862.

6. Winkler, D.,Haltmeier, M.,Kleidorfer, M.,Rauch, W.,Tscheikner-Gratl, F. Pipe failure modelling for water distribution networks using boosted decision trees. Structure and Infrastructure Engineering. 2018, 14(10): 1402-1411.

7. Pegado, R.,Naupari, Z.,Molina, Y.,Castillo, C. Radial distribution network reconfiguration for power losses reduction based on improved selective BPSO. Electric Power Systems Research. 2019, 169: 206-213.

8. Nguyen, T.T.,Nguyen, T.T. An improved cuckoo search algorithm for the problem of electric distribution network reconfiguration. Applied Soft Computing. 2019, 84: 105720-105732.

9. Nguyen, T.T.,Nguyen, T.T.,Truong, A.V.,Nguyen, Q.T.,Phung, T.A. Multi-objective electric distribution network reconfiguration solution using runner-root algorithm. Applied Soft Computing. 2017, 52: 93-108.
10. Atteya, I.I.,Ashour, H.,Fahmi, N.,Strickland, D. Radial distribution network reconfiguration for power losses reduction using a modified particle swarm optimisation. CIRED-Open Access Proceedings Journal. 2017, 2017(1): 2505-2508.

11. Abd Elazim, S.,Ali, E. Improved Whale Optimization Algorithm for Optimal Network Reconfiguration. International Journal of Communications. 2019, 4-9.

12. Jiao, Z.,Ma, K.,Rong, Y.,Wang, P.,Zhang, H.,Wang, S. A path planning method using adaptive polymorphic ant colony algorithm for smart wheelchairs. Journal of Computational Science. 2018, 25: 50-57.

13. Wong, L.A.,Ramachandaramurthy, V.K.,Taylor, P.,Ekanayake, J.,Walker, S.L.,Padmanaban, S. Review on the optimal placement, sizing and control of an energy storage system in the distribution network. Journal of Energy Storage. 2019, 21: 489504.

14. Sun, W.,Harrison, G.P. Wind-solar complementarity and effective use of distribution network capacity. Applied Energy. 2019, 247: 89-101.

15. Abdelaziz, M. Distribution network reconfiguration using a genetic algorithm with varying population size. Electric Power Systems Research. 2017, 142: 911.

16. Oloulade, A.,Imano, A.M.,Vianou, A.,Tamadaho, H. Optimization of the number, size and placement of D-STATCOM in radial distribution network using Ant Colony Algorithm. American Journal of Engineering Research and Reviews. 2018, 1: 12-21.

17. Azizivahed, A.,Arefi, A.,Ghavidel, S.,Shafie-khah, M.,Li, L.,Zhang, J.,Catalão, J.P. Energy management strategy in dynamic distribution network reconfiguration considering renewable energy resources and storage. IEEE Transactions on Sustainable Energy. 2019, 11(2): 662-673. 